\section{US coal plans flout mercury convention}

The first Conference of the Parties (COP1) to the United Nations' Minamata Convention on Mercury will convene next month in Geneva, Switzerland. This is timely in view of the US government's intention to rekindle coal burning for energy. Coal is a major source of mercury pollution, which has well-documented adverse effects on the environment and human health (O. A. Ogunseitan Environ. Sci. Policy Sustain. Dev. 59, 4-13; 2017).

It is challenging to translate multilateral agreements to national policies, as illustrated by the US withdrawal from the Paris climate agreement this year. The United States was the first country to ratify the Minamata Convention. But there are gaps in the alignment of the US domestic agenda with the international framework for pollution prevention that should be high on the agenda for COP1.

The first gap deals with the policy interpretation of scientific uncertainty about mercury's toxicity, with implications for the continuing use of the metal in tooth fillings. The second involves promoting safer alternatives to mercury, including its use in fluorescent lighting. The third and least-understood gap arises from the roles of civic society and transborder institutions in harmonizing domestic and international policies on mercury.

COP1 participants should encourage scientists to educate the public worldwide about the crucial importance of the convention.

Oladele A. Ogunseitan University of California, Irvine, USA. oladele.ogunseitan@uci.edu

\section{Philanthropy research is thriving}

There is much more scientific research about philanthropy than Caroline Fiennes implies (Nature
546, 187; 2017; see also M. L. Stevens Nature 547, 162; 2017).

For example, the Science of Philanthropy Initiative - a collaboration between the University of Chicago in Illinois and the Lilly Family School of Philanthropy in Indiana brings together economists, social psychologists and other practitioners in field experiments and quantitative investigations to gauge what works in philanthropy (see go.nature.com/2x8ymh0).

Hundreds of researchers evaluating different types of philanthropic support are sharing findings through networks such as the Association for Research on Nonprofit Organizations and Voluntary Action, the International Society for ThirdSector Research, and international journals (see also Found. Review http://doi.org/cb25; 2017).

Thanks to the expanding international interest among academics, the science and art of philanthropy are very much alive. Amir Pasic Indiana UniversityPurdue University Indianapolis, Indianapolis, USA. ampasic@iupui.edu

\section{Enlist blockchain to boost conservation}

Blockchain technology stands to improve governance of the environment and of renewable energy (see G. Chapron Nature 545, 403-405; 2017 and

M. Andoni et al. Nature 548, 158; 2017). It could also facilitate peerto-peer fundraising donations to transform nature conservation, which is drastically underfunded.

Cryptocurrency transactions are instant and transparent. They do not require bank accounts and international exchange fees are small. Donations can be sent directly to individuals or projects worldwide, rather than being collected, pooled and distributed by organizations. This encourages donors because the blockchain tracks the impact of donations.

By removing funding barriers, anyone can do conservation work
- from restoring urban ecology to preventing conflicts between humans and wildlife. With a greater number and variety of conservationists and natural spaces, such small participatory efforts will be amplified through social networks.

Peer-to-peer conservation will not solve issues such as the ivory trade, could enable fraudulent or counterproductive projects, and may result in a bias towards flagship species. Yet it can still make an important contribution.

Some 50 years after the launch of the World Wide Fund for Nature, the blockchain now allows us to put a nature fund in every pocket.

Zachary Baynham-Herd University of Edinburgh, UK. z.baynham-herd@ed.ac.uk

\section{Open data settled in materials theory}

Your concerns over impediments to data sharing (Nature 546, 327 ; 2017) are no longer an issue in computational materials science. This is because the Novel Materials Discovery project (NOMAD; https://nomad-coe. eu) has stimulated a cultural shift in attitudes towards open data as a result of the valuable knowledge that has emerged from data mining since early 2014.

NOMAD provides open access to input and output files from the field's major data collections (including http:// aflowlib.org; http://oqmd.org; and https://materialsproject.org), together with those of individual researchers and groups. It contains more than 40 million total-energy calculations, corresponding to billions of core processing hours by highperformance computers globally.

NOMAD hosts the data for a decade or more, offers digital object identifiers (DOIs) to make data citable, and provides services such as an encyclopaedia, big-data analytics tools and advanced graphics.

Hosting the data is expensive, so funding agencies need to step in. However, costs are negligible compared with those of data creation and long-term storage. Claudia Draxl Humboldt University of Berlin, Germany. Francesc Illas University of Barcelona, Spain. Matthias Scheffler Fritz Haber Institute of the Max Planck Society, Berlin, Germany. claudia.draxl@physik.hu-berlin.de

\section{Most lobotomies were done on women}

Our review of the literature on lobotomies in France, Switzerland and Belgium from 1935-85 reveals that the surgical procedure was alarmingly common for female patients ( $84 \%$ of 1,340 subjects). It is not clear whether this reflects a higher prevalence of mental illness among women at the time or their perceived inferior position in those societies, dating from the Napoleonic Code of 1804.

Pioneered by Portuguese neurologist and politician Egas Moniz, lobotomy involves surgery on the brain's prefrontal lobes. He received the Nobel prize in 1949 for the procedure.

Lobotomy is now one of the most highly criticized treatments in history, given its serious effects on the personality. Destructive techniques included classical lobotomy, irradiation with iridium-194, electrocoagulation and intra-cerebral injection of cocaine derivatives.

The treatment of children at this time was particularly deplorable, with 22 children lobotomized for psychomotor agitation to "restore the peace at home" (P. Coquet et al. Pédiatrie 13, 167-173; 1958).

Louis-Marie Terrier Bretonneau Hospital, Tours, France.

Marc Levêque Résidence du Parc Private Hospital, Marseille, France.

Aymeric Amelot La PitiéSalpétrière Hospital, Paris, France. louismarie.terrier@univ-tours.fr 